\title{
Effects of physical therapy-based rehabilitation on recovery of upper limb motor function after stroke in adults: a systematic review and meta-analysis of randomized controlled trials
}

\author{
Jing Huang ${ }^{1 \#}$, Jia-Ri Ji ${ }^{2 \#}$, Chao Liang ${ }^{1}$, Yan-Zhen Zhang ${ }^{1}$, Heng-Cong Sun², Yuan-Hong Yan $^{2}$, \\ Xiao-Bei Xing ${ }^{2}$ \\ ${ }^{1}$ Department of Acupuncture and Moxibustion, Haikou Hospital of Traditional Chinese Medicine, Haikou, China; ${ }^{2}$ Department of Rehabilitation \\ Medicine, Sanya People's Hospital, Sanya, China \\ Contributions: (I) Conception and design: J Huang; (II) Administrative support: XB Xing; (III) Provision of study materials or patients: JR Ji; (IV) \\ Collection and assembly of data: C Liang; (V) Data analysis and interpretation: YZ Zhang; (VI) Manuscript writing: All authors; (VII) Final approval \\ of manuscript: All authors. \\ "These authors contributed equally to this work and should be considered as co-first authors. \\ Correspondence to: Xiao-Bei Xing. Department of Rehabilitation Medicine, Sanya People's Hospital, Sanya 572000, China. \\ Email: xingxiaobei3136@163.com.
}

Backgroundk Limb hemiplegia is one of the common sequelae of stroke. Physical therapy-based rehabilitation training can rapidly improve limb functioning and muscle strength. This study investigated the effect of physical therapy on the recovery of upper limb motor function by employing a literature search and meta-analysis.

Methods: We searched Embase, The Cochrane Central Register of Controlled Trials library, Wiley online library, PubMed, Ovid, and Clinicaltrials.org for randomized controlled trial (RCT) studies and performed an electronic search with the keyword combinations 'physical therapy/rehabilitation' \& 'stroke/post-stroke' \& 'upper extremity/upper limb'. After screening the literature for inclusion criteria and assessing the risk of bias, Revman 5.4 software was used for the analysis and to obtain forest and funnel plots.

Results: A total of 15 RCTs with 1,081 patients were included in the quantitative analysis for this study. The meta-analysis results showed that compared with conventional therapy, physical therapy improved the upper limb Fugl-Meyer Assessment for Upper Extremity (FMA) scores in convalescent stroke patients (MD $=7.27,95 \%$ CI: 4.23-10.32, $\mathrm{Z}=4.68, \mathrm{P}<0.00001)$, and increased the Functional Independence Measure (FIM) scores $(\mathrm{MD}=18.82$, 95\% CI: 6.34-31.30, $\mathrm{Z}=2.96, \mathrm{P}=0.003)$, the Action Research Arm Test (ARAT) scores $(\mathrm{MD}=8.84,95 \%$ CI: 6.53-11.15, $\mathrm{Z}=7.50, \mathrm{P}<0.00001)$, and the Box and Block Test (BBT) scores $(\mathrm{MD}=6.11$, 95\% CI: $1.18-11.04, \mathrm{Z}=2.43, \mathrm{P}=0.02$ ) of patients during the rehabilitation period.

Discussion: The use of physical therapy-based rehabilitation training in the recovery period for stroke patients with hemiplegia can improve upper limb movement ability, increase muscle strength, reduce limb pain, and improve the quality of life.

Keywords: Physical therapy; rehabilitation training; stroke; upper limb motor function; meta-analysis

Submitted Nov 17, 2021. Accepted for publication Jan 30, 2022.

doi: 10.21037/apm-21-3710

View this article at: https://dx.doi.org/10.21037/apm-21-3710 


\section{Introduction}

Stroke is a common disease in neurology, with disability and mortality rates as high as $80 \%$ (1). The middle-aged and the elderly are the high incidence population of stroke. The function of tissues and organs of the elderly is declining, the autonomic nerve activity is disordered, the ability to regulate vasomotor and systolic is reduced, the degree of vascular wall sclerosis is aggravated, and the possibility of ischemic stroke is increased (2). After rescuing from death stroke patients nearly always have some kind of sequelae, such as coma, expression disorder, sensory disturbance, and limb hemiplegia, which create a self-care ability decline and affect their quality of life (3). Limb hemiplegia is a common sequela of stroke where patients present with loss of muscle strength and inability to perform free limb movements $(4,5)$. Clinically, the primary treatments for hemiplegia after stroke include drugs and physical therapy. The drugs are mainly used to scavenge free radicals, improve brain function, and promote the recovery of limb function. Rehabilitation training can rapidly improve limb functioning and muscle strength, prevent muscle atrophy and joint stiffness, reduce the disability rate, and improve patients' quality of life (6). Many different physical therapies are used clinically (7-9), and systematic analysis of these therapies is always focusing on just one kind of therapy. This study used a meta-analysis to quantitatively assess the effect of various physical therapy on limb hemiplegia during stroke rehabilitation. We present the following article in accordance with the PRISMA reporting checklist (available at https://apm.amegroups.com/ article/view/10.21037/apm-21-3710/rc).

\section{Methods}

\section{Database and search strategy}

We searched Embase, The Cochrane Central Register of Controlled Trials library, Wiley online library, PubMed, and Ovid, as well as Clinicaltrials.org for clinical trial studies (clinical experimental research). We set the search time from the establishment of the databases to September 2021 and performed the electronic search with the keyword combinations 'physical therapy/rehabilitation' \& 'stroke/ post-stroke' \& 'upper extremity/upper limb'.

\section{Inclusion criteria}

(I) Study types: we included English language articles of randomized controlled trial (RCT) clinical studies. (II)
Study subjects: all patients were upper limb hemiplegic patients in the recovery period after acute stroke (whether ischemic or hemorrhagic stroke), and regardless of whether the hemiplegic site was on the left or right side. (III) The experimental group intervention measures used physical therapy as the primary rehabilitation method (limb movement, visual imagery induction, music relaxation, acupuncture, electrical nerve stimulation, etc.), and a control group was included that received conventional care or therapy. If there were other treatment or control groups, these were not included in the analysis. The experimental and control groups received the same drug and other basic treatments. (IV) At the very least, studies were required to contain an upper limb rehabilitation index and the ability to provide complete data.

\section{Exclusion criteria}

(I) We excluded patients with chronic stroke; (II) interventions using combined therapies (where the physical therapy was not described) were excluded in addition to any studies that did not describe the intervention frequency and intervention methods in detail; (III) investigations, case studies, and reviews of non-randomized controlled trials were excluded, as well as pilot studies; (IV) articles with missing rehabilitation indicators or data that could not be transformed and used were also excluded.

\section{Literature screening}

After the literature retrieval, all articles were imported into Endnote X9 software. Duplicate articles were excluded by the software. Two researchers independently read the titles and abstracts for further screening. If the original text could not be obtained from the internet, the author of the original text was contacted.

\section{Literature quality evaluation and risk-of-bias evaluation}

The Cochrane Handbook for Systematic Reviews of Interventions (Cochrane Collaboration) was used to evaluate the risk of bias in the included trials. The six aspects evaluated in the trials were as follows: (I) random allocation method; (II) blinding method; (III) implementation of allocation concealment; (IV) data integrity; (V) selective reporting bias; (VI) other biases. These six aspects were evaluated as either "low risk", "unclear", or "high risk". If a study was evaluated as low risk in all six aspects, it was rated 
as Level A; if there were one or more "unclear" evaluations, it was rated as Level B; if there were one or more "high risk" evaluations, it was rated as Level C.

\section{Outcome indicators}

Because there are many efficacy indicators of upper limb recovery, we only counted the four most common indicators: (I) the Fugl-Meyer Assessment for Upper Extremity (FMA-UE); (II) the Functional Independence Measure (FIM); (III) the Action Research Arm Test (ARAT); (IV) the Box and Block Test (BBT).

\section{Data extraction}

Two researchers independently extracted the data. The extracted data included title, author, publication year and month, grouping method, number of cases in each group, patient age, gender ratio, stroke time, primary hemiplegic side, intervention measures, observation time, followup time, and rehabilitation indicators. Following the data extraction, the two researchers cross-checked each other's results, and any differences of opinion were resolved by discussion.

\section{Statistical methods}

(I) RevMan 5.4 software (released by The Cochrane Collaboration) was used for the meta-analysis; (II) inverse variance statistics were used for the continuous variables, and the mean difference (MD) and $95 \%$ confidence interval (CI) were used to report the statistics where $\mathrm{P}<0.05$ indicated a statistically significant difference; (III) forest plot descriptive statistics were used to display the results; (IV) the $\mathrm{I}^{2}$ analysis and Q test were used to assess the degree of heterogeneity amongst studies. An $\mathrm{I}^{2}$ value $>50 \%$ or a $\mathrm{P}$ value $<0.1$ was used to indicate significant heterogeneity, in which case a random-effects model was used to obtain the odds ratio (OR) value; otherwise, a fixed-effects model was used to obtain the OR value; $(V)$ if the heterogeneity analysis suggested heterogeneity between studies, a case-bycase exclusion method was used to investigate the source of the heterogeneity. A general description was adopted when the source of the heterogeneity could not be identified; (VI) a sensitivity analysis was performed by comparing the results of the random and fixed effects; and (VII) a funnel plot was used to display any potential publication bias.

\section{Results}

\section{Literature screening results}

Figure 1 illustrates the flow chart of the literature selection, where a final 15 studies with a total of 1,081 patients were included in the quantitative analysis. A list of the basic characteristics, intervention measures, and rehabilitation indicators for all studies is shown in Table 1.

\section{Literature quality and bias evaluation}

In this study, all included RCTs (10-24) described the method of random sequence generation (using the permuted block randomization method or the computer random sequence generation method). Two studies $(14,22)$ failed to describe the allocation concealment method, and the remainder used the sealed opaque envelope concealment method. Only two studies $(16,22)$ failed to describe the blinding implementation method. All of the RCTs recorded dropout cases in detail, without any obvious selective reporting bias or other biases, and the overall quality was excellent, as shown in Table 2.

\section{Meta-analysis results}

Effect of physical therapy on the upper limb FMA index (FMA-UE)

Excepting two studies $(13,23)$, a total of 13 studies reported FMA scores following the intervention. Statistical heterogeneity was evident in the FMA scores $\left(\mathrm{I}^{2}=68 \%\right.$, $\mathrm{P}=0.0002$ ), so a random-effects model was used. Metaanalysis showed that physical therapy improved the upper limb FMA scores of stroke patients in the convalescent stage ( $\mathrm{MD}=7.27,95 \% \mathrm{CI}: 4.23-10.32, \mathrm{Z}=4.68, \mathrm{P}<0.00001$ ), as shown in Figure 2.

The studies were also divided according to the type of physical therapy intervention; a virtual reality game group, a constraint-induced movement (CIM) therapy group, a mirror therapy group, and a robotic-assisted therapy group. The results showed that there was no heterogeneity in the subgroup data. The meta-analysis results for each group are shown in Figure 2.

\section{Effect of physical therapy on the FIM index}

A total of three articles $(10,13,17)$ reported the FIM index following intervention, with statistical heterogeneity evident between the articles $\left(\mathrm{I}^{2}=76 \%, \mathrm{P}=0.02\right)$. Using the random- 


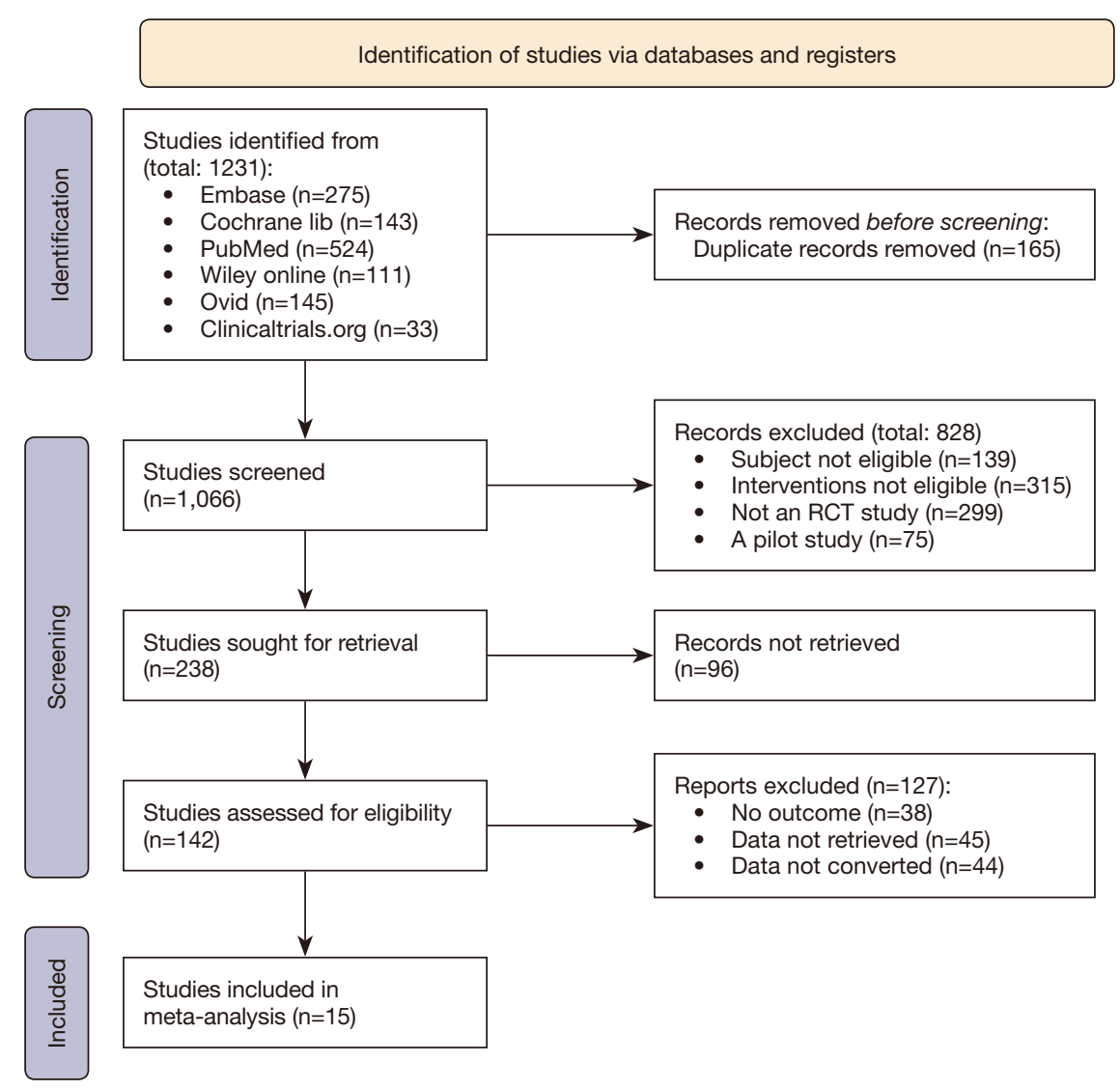

Figure 1 Literature selection flow chart.

effects model, the meta-analysis results showed that physical therapy increased the FIM score of patients during the rehabilitation period (MD =18.82, 95\% CI: 6.34-31.30, $\mathrm{Z}=2.96, \mathrm{P}=0.003$ ), as shown in Figure 3.

\section{Effect of physical therapy on the ARAT index}

Three articles $(13,14,16)$ reported the ARAT index scores, with no statistical heterogeneity evident between them $\left(\mathrm{I}^{2}=0 \%, \mathrm{P}=0.73\right)$. Meta-analysis showed that physical therapy increased the ARAT score during the rehabilitation period ( $\mathrm{MD}=8.84,95 \% \mathrm{CI}: 6.53-11.15, \mathrm{Z}=7.50, \mathrm{P}<0.00001)$, as shown in Figure 4.

\section{Effect of physical therapy on BBT index scores}

Three articles $(17,21,23)$ reported BBT scores, with no statistical heterogeneity evident among them $\left(\mathrm{I}^{2}=0 \%\right.$, $\mathrm{P}=0.69)$. Using a fixed-effects model, the meta-analysis results indicated that physical therapy increased the BBT score of patients during the rehabilitation period $(\mathrm{MD}=6.11$, 95\% CI: 1.18-11.04, Z=2.43, P=0.02), as shown in Figure 5 .

\section{Source of heterogeneity and sensitivity analysis}

In the analysis of FMA indicators, there was statistical heterogeneity among the results of the studies $\left(\mathrm{I}^{2}=68 \%\right.$, $\mathrm{P}=0.0002)$. However, when the studies were divided into four subgroups according to the intervention method, there was no heterogeneity, indicating that the intervention method was the largest source of heterogeneity. The analyses of the ARAT and BBT scores showed no significant differences in the results between the random- and fixedeffects models, indicating that the results had good stability (good sensitivity).

\section{Analysis of publication bias}

The funnel plot of the meta-analysis for the FMA-UE scores shows that the two sides of the funnel are almost symmetrical, suggesting that the publication bias is small (see Figure 6).

\section{Discussion}

Stroke is very common in the elderly population, and 


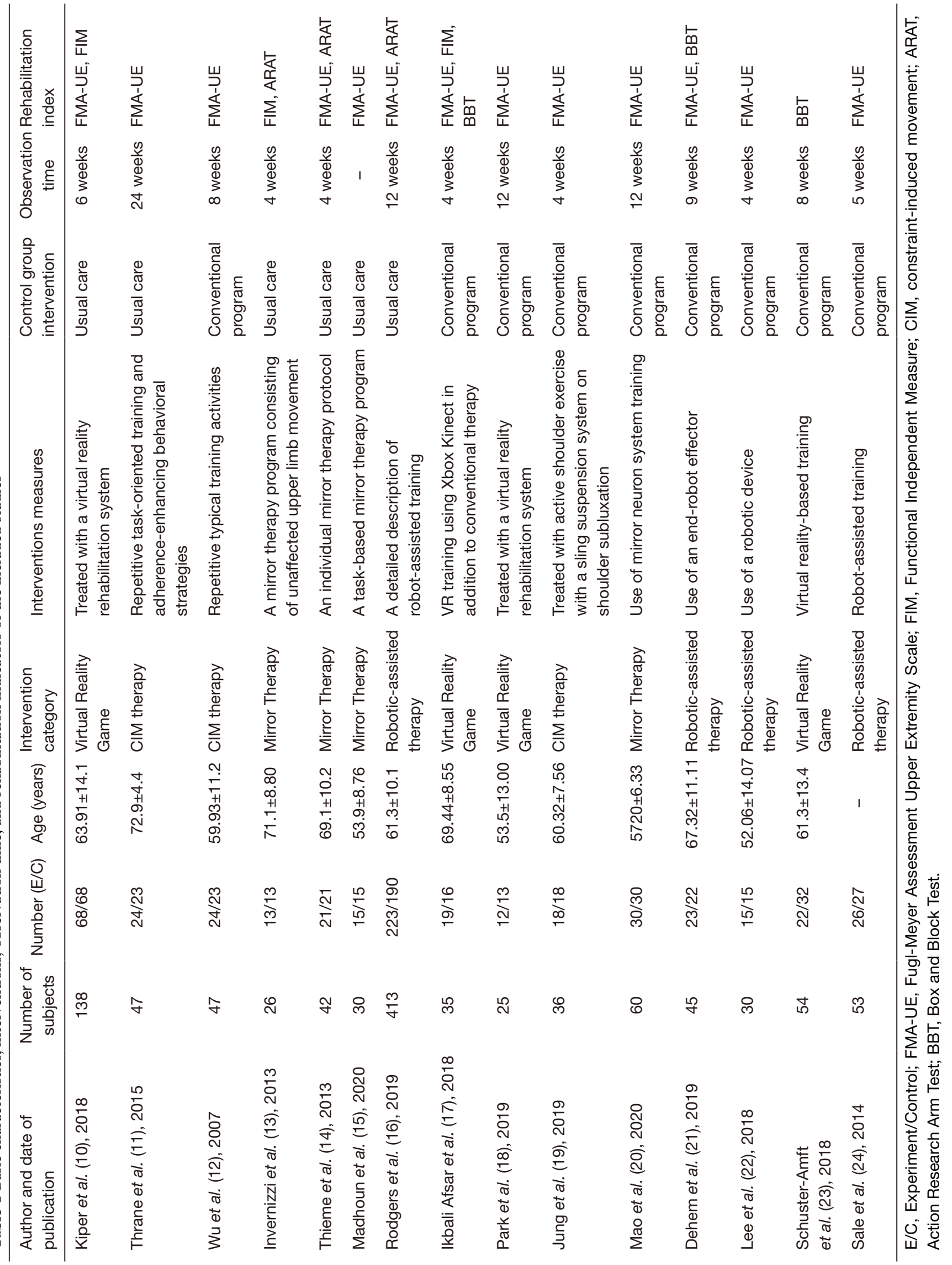


Table 2 Risk of bias and quality assessment based on the Cochrane Handbook for Evaluation of Randomized Interventions

\begin{tabular}{|c|c|c|c|c|c|c|c|}
\hline Study & $\begin{array}{c}\text { Random sequence } \\
\text { generation }\end{array}$ & $\begin{array}{c}\text { Classification } \\
\text { Hiding }\end{array}$ & Blind method & Data integrity & $\begin{array}{l}\text { Optional } \\
\text { reporting }\end{array}$ & Other bias & Grade \\
\hline Kiper et al. (10), 2018 & Low & Low & Low & Low & Low & Low & Class $\mathrm{A}$ \\
\hline Thrane et al. (11), 2015 & Low & Low & Low & Low & Low & Low & Class $\mathrm{A}$ \\
\hline Wu et al. (12), 2007 & Low & Low & Low & Low & Low & Low & Class $\mathrm{A}$ \\
\hline Madhoun et al. (15), 2020 & Low & Low & Low & Low & Low & Low & Class $\mathrm{A}$ \\
\hline Rodgers et al. (16), 2019 & Low & Low & Unclear & Low & Low & Low & Class B \\
\hline Ikbali Afsar et al. (17), 2018 & Low & Low & Low & Low & Low & Low & Class A \\
\hline Dehem et al. (21), 2019 & Low & Low & Low & Low & Low & Low & Class A \\
\hline Lee et al. (22), 2018 & Low & Unclear & Unclear & Low & Low & Low & Class B \\
\hline Schuster-Amft et al. (23), 2018 & Low & Low & Low & Low & Low & Low & Class $\mathrm{A}$ \\
\hline Sale et al. (24), 2014 & Low & Low & Low & Low & Low & Low & Class $\mathrm{A}$ \\
\hline
\end{tabular}

hemiplegia is the main sequela after stroke, accounting for approximately $50-70 \%$ of all sequelae (25). In the recovery period after acute stroke, physical rehabilitation therapy can gradually reactivate the neuronal cells in the patient's brain by squeezing, weight-bearing, traction, stimulation, and other movement methods. These activities promote muscle contraction and improve muscle strength so that the function of the hemiplegic limbs can be recovered (26). In this study, 15 high-quality RCT studies with a total of 1,081 participants were included. The results showed that physical therapy-based rehabilitation training significantly improved the upper limb function assessment scores, thereby not only improving the symptoms of limb hemiplegia but also the mobility and quality of life for stroke patients. After stroke, the balance of mutual influence and restriction between the two cerebral hemispheres is broken. Due to the denervation of the lesion area of the damaged cerebral hemisphere, the ability to receive and transmit descending motor signals is much lower than before, and the motor function of controlling limbs is weakened. At this time, the affected cerebral nerve will also be inhibited and interfered by the opposite neurons from the healthy cerebral hemisphere through the corpus callosum, resulting in further inhibition of the repair of the damaged cerebral nerve on the affected side and affecting the recovery of limb motor function on the affected side. The rehabilitation training based on physical therapy reduces the mutual inhibition between cerebral hemispheres, helping patients recover limb function as soon as possible (27).

In this study, the articles were divided into four subgroups according to the different interventions: a virtual reality game group, a CIM therapy group, a mirror therapy group, and a robot-assisted therapy group. Meta-analysis showed that the upper limb function assessment scores of the different interventions were higher than those of the control group. Virtual reality technology involves using computer-based programs designed to simulate real-life objects and events by using virtual interactions and feedback provided through the senses, such as vision, hearing, touch, smell, and movement. Patients complete the task training and repetitive training in the virtual environment and complete physical exercise therapy under the premise of ensuring safety (28). Mirror therapy reproduces an inverse image of the unaffected limb to allow the patient to imagine movement and perform rehabilitation training through a combination of visual illusion and visual feedback (29). CIM therapy constrains the movement of the healthy upper limb while requiring mandatory use of the hemiplegic upper limb 


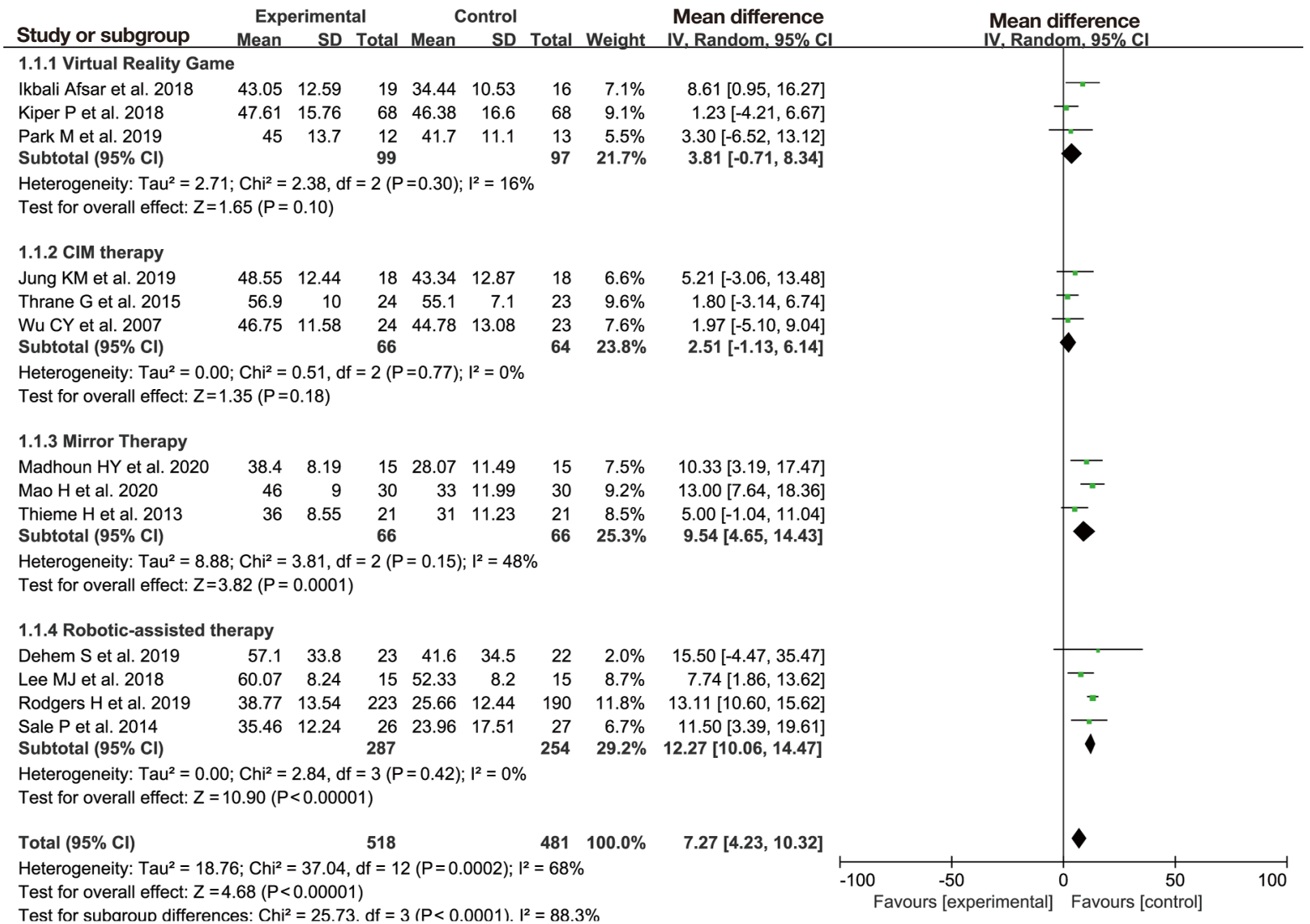

Figure 2 Effect of physical therapy on FMA-UE scores of stroke patients during rehabilitation. FMA-UE, Fugl-Meyer Assessment Upper Extremity Scale.

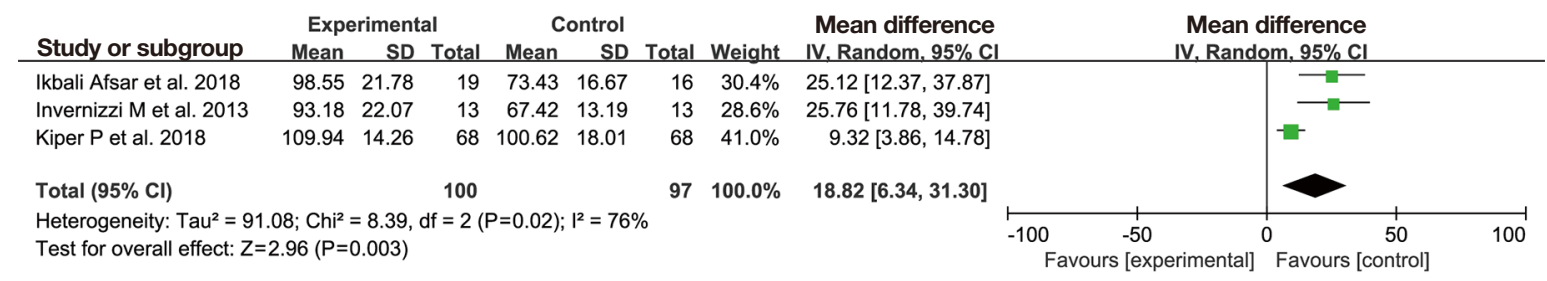

Figure 3 Effect of physical therapy on FIM scores of stroke patients during rehabilitation. FIM, Functional Independent Measure.

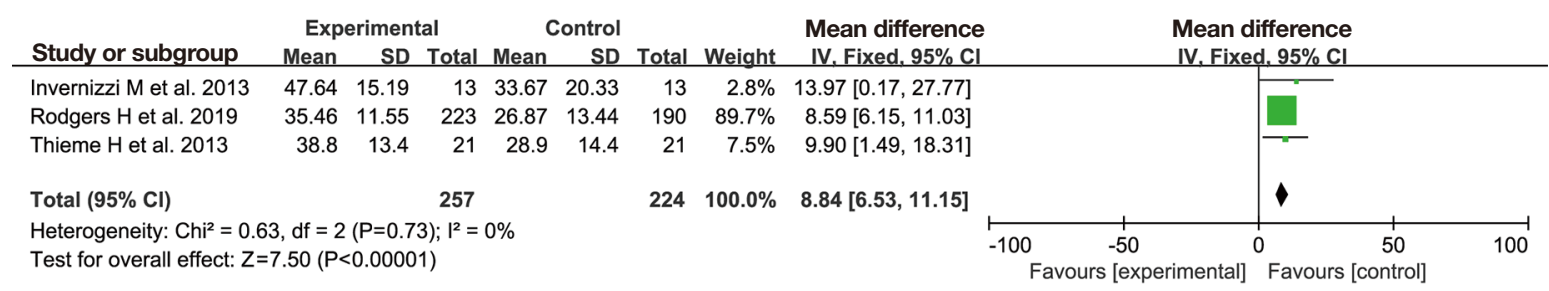

Figure 4 Effect of physical therapy on ARAT scores of stroke patients during rehabilitation. ARAT, Action Research Arm Test. 


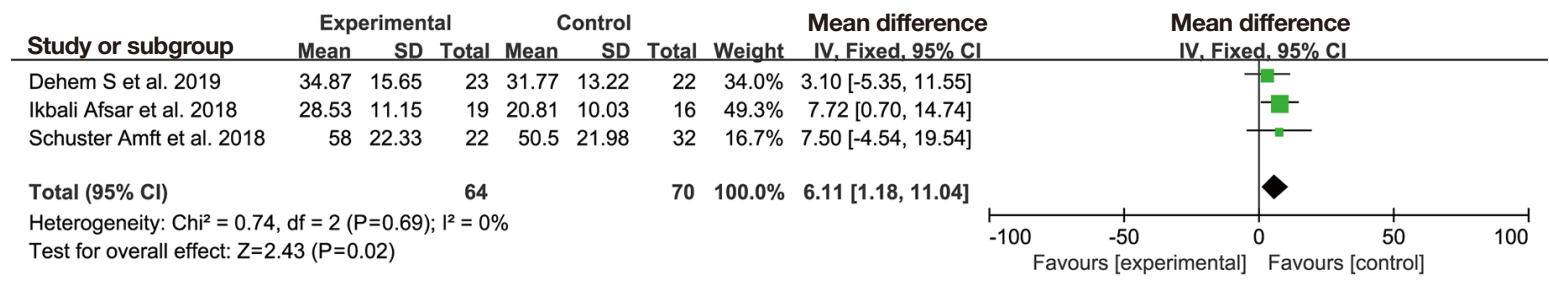

Figure 5 Effect of physical therapy on BBT scores of stroke patients during rehabilitation. BBT, Box and Block Test.

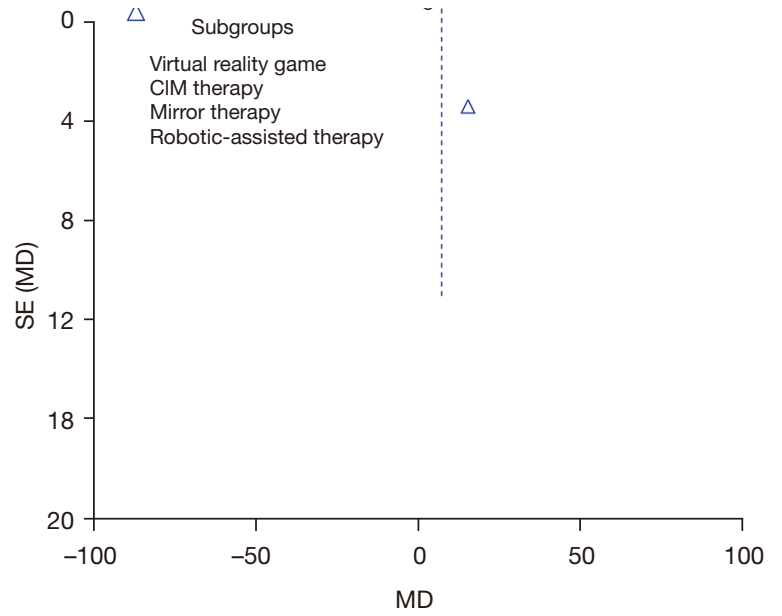

Figure 6 Funnel plot of the effect of physical therapy on FMA-UE scores of stroke patients during rehabilitation. FMA-UE, FuglMeyer Assessment Upper Extremity Scale.

for the rehabilitation techniques (30). Due to the advances in artificial intelligence control technology in recent years, robot-assisted therapy is now being applied in rehabilitation therapy for stroke patients. Robot-assisted therapy can aid in the active movement, passive traction, and resistance movement of the patient's limbs by using robotic devices and provides visual and auditory feedback, thus providing a more motivating and enjoyable experience of rehabilitation training for the patient (31).

Study (32) has suggested that improving exercise intensity enables better patient outcomes. However, we believe that although physical therapy-based rehabilitation training can benefit patients, the training process still needs to be performed under the guidance of a physiotherapist.

Although combination therapy $(33,34)$ may achieve better efficacy than single physical therapy, we did not include combination therapy in the study, given the heterogeneity that may arise. A previous study (35) reported that physical therapy also benefited hemiplegia in chronic stroke patients, but given the potential for heterogeneity, we did not include chronic stroke patients in this study and only included patients in the recovery period after acute stroke. In addition, some studies $(36,37)$ have shown that electrical nerve stimulation treatment also has a good effect, but due to the different outcome measures used, no relevant studies were included in the present meta-analysis. Action observation therapy (AOT) is a new method grounded on neuroscience and mirror neuron system which is the basis of mirror therapy, too. This method requires patients to form accurate motion images in their minds, learn and practice actions after observing behaviors and actions, so as to restore cognitive activity and limb movement (38). However, there are few studies on this method, and we have not included any literature, which may become the research direction in the future.

In addition, patients of different ages and educational levels have different needs for rehabilitation training guidance and rehabilitation training knowledge publicity, and their compliance in the process of rehabilitation training also different. Patients with younger age and higher educational level have higher needs for rehabilitation training guidance and knowledge, while patients with older age need detailed guidance, in the meanwhile correcting the depressive state of mind (39).

In this study, the primary source of heterogeneity between studies arose from the different types of physical therapy used. The funnel plot showed a symmetrical distribution, suggesting that the publication bias was negligible. Although the quality of the studies included in this meta-analysis was high, the sample size was relatively small. Therefore, future research should consist of RCTs with larger sample sizes to comprehensively explore this topic. 


\section{Conclusions}

A total of 1,081 patients were included in 15 studies in this meta-analysis. The results showed that physical therapybased rehabilitation training during the recovery period of stroke patients with hemiplegia improves upper limb activity, increases muscle strength, reduces limb pain, and improves the quality of life. However, due to the small number of articles included in this study, further RCTs with larger sample sizes are required to comprehensively explore this area of research.

\section{Acknowledgments}

Funding: This work was funded by the Hainan Health Statistical Industry Research Project, China (19A200100) supported by Hainan Province Clinical Medical Center.

\section{Footnote}

Reporting Checklist: The authors have completed the PRISMA reporting checklist. Available at https://apm. amegroups.com/article/view/10.21037/apm-21-3710/rc

Conflicts of Interest: All authors have completed the ICMJE uniform disclosure form (available at https://apm. amegroups.com/article/view/10.21037/apm-21-3710/coif). The authors have no conflicts of interest to declare.

Ethical Statement: The authors are accountable for all aspects of the work in ensuring that questions related to the accuracy or integrity of any part of the work are appropriately investigated and resolved.

Open Access Statement: This is an Open Access article distributed in accordance with the Creative Commons Attribution-NonCommercial-NoDerivs 4.0 International License (CC BY-NC-ND 4.0), which permits the noncommercial replication and distribution of the article with the strict proviso that no changes or edits are made and the original work is properly cited (including links to both the formal publication through the relevant DOI and the license). See: https://creativecommons.org/licenses/by-nc-nd/4.0/.

\section{References}

1. Raghavan P. Upper Limb Motor Impairment After Stroke. Phys Med Rehabil Clin N Am 2015;26:599-610.
2. Coupar F, Pollock A, Rowe P, et al. Predictors of upper limb recovery after stroke: a systematic review and metaanalysis. Clin Rehabil 2012;26:291-313.

3. Marque P, Gasq D, Castel-Lacanal E, et al. Post-stroke hemiplegia rehabilitation: evolution of the concepts. Ann Phys Rehabil Med 2014;57:520-9.

4. Griffin C. Management of the hemiplegic shoulder complex. Top Stroke Rehabil 2014;21:316-8.

5. Byrd EM, Jablonski RJ, Vance DE. Understanding Anosognosia for Hemiplegia After Stroke. Rehabil Nurs 2020;45:3-15.

6. Paci M. Physiotherapy based on the Bobath concept for adults with post-stroke hemiplegia: a review of effectiveness studies. J Rehabil Med 2003;35:2-7.

7. Veerbeek JM, Langbroek-Amersfoort AC, van Wegen EE, et al. Effects of Robot-Assisted Therapy for the Upper Limb After Stroke. Neurorehabil Neural Repair 2017;31:107-21.

8. Hong Z, Sui M, Zhuang Z, et al. Effectiveness of Neuromuscular Electrical Stimulation on Lower Limbs of Patients With Hemiplegia After Chronic Stroke: A Systematic Review. Arch Phys Med Rehabil 2018;99:10111022.e1.

9. Tomida K, Sonoda S, Hirano S, et al. Randomized Controlled Trial of Gait Training Using Gait Exercise Assist Robot (GEAR) in Stroke Patients with Hemiplegia. J Stroke Cerebrovasc Dis 2019;28:2421-8.

10. Kiper P, Szczudlik A, Agostini M, et al. Virtual Reality for Upper Limb Rehabilitation in Subacute and Chronic Stroke: A Randomized Controlled Trial. Arch Phys Med Rehabil 2018;99:834-842.e4.

11. Thrane G, Askim T, Stock R, et al. Efficacy of Constraint-Induced Movement Therapy in Early Stroke Rehabilitation: A Randomized Controlled Multisite Trial. Neurorehabil Neural Repair 2015;29:517-25.

12. Wu CY, Chen CL, Tang SF, et al. Kinematic and clinical analyses of upper-extremity movements after constraintinduced movement therapy in patients with stroke: a randomized controlled trial. Arch Phys Med Rehabil 2007;88:964-70.

13. Invernizzi $M$, Negrini $S$, Carda $S$, et al. The value of adding mirror therapy for upper limb motor recovery of subacute stroke patients: a randomized controlled trial. Eur J Phys Rehabil Med 2013;49:311-7.

14. Thieme H, Bayn M, Wurg M, et al. Mirror therapy for patients with severe arm paresis after stroke--a randomized controlled trial. Clin Rehabil 2013;27:314-24.

15. Madhoun HY, Tan B, Feng Y, et al. Task-based mirror 
therapy enhances the upper limb motor function in subacute stroke patients: a randomized control trial. Eur J Phys Rehabil Med 2020;56:265-71.

16. Rodgers H, Bosomworth H, Krebs HI, et al. Robot assisted training for the upper limb after stroke (RATULS): a multicentre randomised controlled trial. Lancet 2019;394:51-62.

17. Ikbali Afsar S, Mirzayev I, Umit Yemisci O, et al. Virtual Reality in Upper Extremity Rehabilitation of Stroke Patients: A Randomized Controlled Trial. J Stroke Cerebrovasc Dis 2018;27:3473-8.

18. Park M, Ko MH, Oh SW, et al. Effects of virtual realitybased planar motion exercises on upper extremity function, range of motion, and health-related quality of life: a multicenter, single-blinded, randomized, controlled pilot study. J Neuroeng Rehabil 2019;16:122.

19. Jung KM, Choi JD. The Effects of Active Shoulder Exercise with a Sling Suspension System on Shoulder Subluxation, Proprioception, and Upper Extremity Function in Patients with Acute Stroke. Med Sci Monit 2019;25:4849-55.

20. Mao H, Li Y, Tang L, et al. Effects of mirror neuron system-based training on rehabilitation of stroke patients. Brain Behav 2020;10:e01729.

21. Dehem S, Gilliaux M, Stoquart G, et al. Effectiveness of upper-limb robotic-assisted therapy in the early rehabilitation phase after stroke: A single-blind, randomised, controlled trial. Ann Phys Rehabil Med 2019;62:313-20.

22. Lee MJ, Lee JH, Lee SM. Effects of robot-assisted therapy on upper extremity function and activities of daily living in hemiplegic patients: A single-blinded, randomized, controlled trial. Technol Health Care 2018;26:659-66.

23. Schuster-Amft C, Eng K, Suica Z, et al. Effect of a fourweek virtual reality-based training versus conventional therapy on upper limb motor function after stroke: A multicenter parallel group randomized trial. PLoS One 2018;13:e0204455.

24. Sale P, Franceschini M, Mazzoleni S, et al. Effects of upper limb robot-assisted therapy on motor recovery in subacute stroke patients. J Neuroeng Rehabil 2014;11:104.

25. Borges LR, Fernandes AB, Melo LP, et al. Action observation for upper limb rehabilitation after stroke. Cochrane Database Syst Rev 2018;10:CD011887.

26. Pollock A, Farmer SE, Brady MC, et al. Interventions for improving upper limb function after stroke. Cochrane Database Syst Rev 2014;(11):CD010820.

27. Laver KE, Lange B, George S, et al. Virtual reality for stroke rehabilitation. Cochrane Database Syst Rev 2017;11:CD008349.

28. Rossiter HE, Borrelli MR, Borchert RJ, et al. Cortical mechanisms of mirror therapy after stroke. Neurorehabil Neural Repair 2015;29:444-52.

29. Pérez-Cruzado D, Merchán-Baeza JA, GonzálezSánchez M, et al. Systematic review of mirror therapy compared with conventional rehabilitation in upper extremity function in stroke survivors. Aust Occup Ther J 2017;64:91-112.

30. Corbetta D, Sirtori V, Castellini G, et al. Constraintinduced movement therapy for upper extremities in people with stroke. Cochrane Database Syst Rev 2015;(10):CD004433.

31. Bertani R, Melegari C, De Cola MC, et al. Effects of robot-assisted upper limb rehabilitation in stroke patients: a systematic review with meta-analysis. Neurol Sci 2017;38:1561-9.

32. Bang DH. Effect of Modified Constraint-Induced Movement Therapy Combined with Auditory Feedback for Trunk Control on Upper Extremity in Subacute Stroke Patients with Moderate Impairment: Randomized Controlled Pilot Trial. J Stroke Cerebrovasc Dis 2016;25:1606-12.

33. Kim H, Yoo EY, Jung MY, et al. The effects of mental practice combined with modified constraint-induced therapy on corticospinal excitability, movement quality, function, and activities of daily living in persons with stroke. Disabil Rehabil 2018;40:2449-57.

34. Kim JH, Lee BH. Mirror therapy combined with biofeedback functional electrical stimulation for motor recovery of upper extremities after stroke: a pilot randomized controlled trial. Occup Ther Int 2015;22:51-60.

35. Colomer C, NOé E, Llorens R. Mirror therapy in chronic stroke survivors with severely impaired upper limb function: a randomized controlled trial. Eur J Phys Rehabil Med 2016;52:271-8.

36. Kim SH, Park JH. The Effect of Occupation-Based Bilateral Upper Extremity Training in a Medical Setting for Stroke Patients: A Single-Blinded, Pilot Randomized Controlled Trial. J Stroke Cerebrovasc Dis 2019;28:104335.

37. Du J, Yang F, Hu J, et al. Effects of high- and lowfrequency repetitive transcranial magnetic stimulation on motor recovery in early stroke patients: Evidence from a randomized controlled trial with clinical, neurophysiological and functional imaging assessments. Neuroimage Clin 2019;21:101620. 
38. Shih TY, Wu CY, Lin KC, et al. Effects of action observation therapy and mirror therapy after stroke on rehabilitation outcomes and neural mechanisms by MEG: study protocol for a randomized controlled trial. Trials 2017;18:459.

39. de Menezes KKP, Ada L, Teixeira-Salmela LF, et al. Home-
Based Interventions may Increase Recruitment, Adherence, and Measurement of outcomes in Clinical Trials of Stroke Rehabilitation. J Stroke Cerebrovasc Dis 2021;30:106022.

(English Language Editor: D. Fitzgerald)

Cite this article as: Huang J, Ji JR, Liang C, Zhang YZ, Sun HC, Yan YH, Xing XB. Effects of physical therapy-based rehabilitation on recovery of upper limb motor function after stroke in adults: a systematic review and meta-analysis of randomized controlled trials. Ann Palliat Med 2022;11(2):521-531. doi: 10.21037/apm-21-3710 